\title{
Establishment of a Host-to-Host Transmission Model for Mycobacterium avium subsp. hominissuis Using Caenorhabditis elegans and Identification of Colonization-Associated Genes
}

\author{
Luiz E. Bermudez ${ }^{1,2 *}$, Sasha J. Rose ${ }^{1,2}$, Jamie L. Everman ${ }^{1,2 \dagger}$ and Navid R. Ziaie ${ }^{1}$ \\ ${ }^{1}$ Department of Biomedical Sciences, College of Veterinary Medicine, Oregon State University, Corvallis, OR, United States, \\ ${ }^{2}$ Department of Microbiology, College of Science, Oregon State University, Corvallis, OR, United States
}

OPEN ACCESS

Edited by:

Kathleen McDonough, Wadsworth Center, United States

Reviewed by:

Raul G. Barletta,

University of Nebraska-Lincoln,

United States

Chang-Shi Chen,

National Cheng Kung University,

Taiwan

${ }^{*}$ Correspondence:

Luiz E. Bermudez

luiz.bermudez@oregonstate.edu

tPresent Address:

Jamie L. Everman,

National Jewish Health, Denver, CO,

United States

Received: 27 January 2018 Accepted: 05 April 2018 Published: 24 April 2018

Citation:

Bermudez LE, Rose SJ, Everman JL and Ziaie NR (2018) Establishment of a Host-to-Host Transmission Model for Mycobacterium avium subsp. hominissuis Using Caenorhabditis elegans and Identification of

Colonization-Associated Genes.

Front. Cell. Infect. Microbiol. 8:123.

doi: 10.3389/fcimb.2018.00123
Mycobacterium avium subsp. hominissuis ( $M$. avium) is a member of the non-tuberculous mycobacteria (NTM), and is a common cause of lung infection in patients with chronic NTM lung conditions. $M$. avium is an environmental bacterium believed to be transmitted from environmental sources. In this work we used a recently developed model in Caenorhabditis elegans to ask whether M. avium can be transmitted from host-to-host, and the bacterial genes associated with host colonization. Infection of $C$. elegans was carried out by placing the nematode in cultured with $M$. avium. Bacteria eliminated from the intestines of infected $C$. elegans were used to infect naïve nematodes. In parallel experiments, to identify colonization associated genes, a transposon library of $M$. avium was screened for the ability to bind to HEp-2 mucosal cells. Thirty clones were identified and five selected clones with impaired adherence to HEp-2 epithelial cells were used to infect $C$. elegans to determine the degree of colonization. It was determined that $M$. avium eliminated from infected $C$. elegans were able to colonize a naïve $C$. elegans with high efficiency. Thirty of the most adherence-deficient $M$. avium clones obtained from the HEp-2 cell screening were sequenced to identify the location of the transposon. Many of the genes associated with the bacterial cell wall synthesis were shown to be inactivated in the selected mutants. Five out of the 30 bacterial clones were then used to infect C. elegans. All five mutants had impaired ability to colonize $C$. elegans compared with the wild type bacteria (decrease of 1.5-2.0 logs, $p<0.05$ ). The limitation of this work is that the model can be used for initial screening, but other more complex systems should be used to confirm the findings. C. elegans can be used as a model to test for $M$. avium adherence/colonization-associated virulence determinants. All the tested adherence-deficient clones that were examined had impaired ability to colonize the host C. elegans, and some can be potentially used to prevent colonization.

\footnotetext{
Keywords: $M$. avium, transmission, host-to-host, colonization genes, C. elegans
} 


\section{INTRODUCTION}

Infections caused by non-tuberculous mycobacteria (NTM) are becoming increasingly common worldwide (Primm et al., 2004; Falkinham, 2009). Individuals with chronic lung diseases, such as emphysema, bronchiectasis, and cystic fibrosis have enhanced susceptibility to infections caused by Mycobacterium avium subsp. hominissuis and Mycobacterium abscessus (Griffith et al., 2007; Ringshausen et al., 2016). M. avium subsp. hominissuis, is the most commonly isolate obtained from patients with lung disease, although the pathogen is also seen associated with disseminated infections in patients with AIDS and other immunosuppressive conditions (Faria et al., 2015; Ringshausen et al., 2016). Options for treatment of NTM infections are limited mainly as a consequence of the intrinsic resistance of this group of microorganisms to the majority of the currently available antibiotics, a fact amplified by the absence of drug discovery programs (Adjemian et al., 2012).

Because NTM are generally environmental microorganisms, it has been assumed that exposure to environmental conditions would be the most common form of transmission to the host (Namkoong et al., 2016). The fact, however, as with many infectious diseases, transmission models are difficult to develop, which creates limitations with the ability to investigate and ultimately understand how pathogens spread among hosts. In addition, to prove that an environmental infection can also be transmitted from host-to-host, and to examine strategies that can be used to prevent infections, is definitively challenging.

Recently, an outbreak of NTM caused by M. abscessus in an intensive care unit brought up the importance of the transmission of NTM by other means that differ from the accepted environmental sources (Vaghaiwalla et al., 2014). The numerous or perhaps the prolonged exposure to infected individuals may lead to the transmission of NTMs to other individuals, however, the possibility of developing disease is strongly connected to the immune status of the host and probably the duration of contact (Jamal et al., 2014). Therefore, epidemiologically, it is quite difficult to establish the link between the source of transmission and infection (or disease), with the rare exceptions of the few outbreaks, in which an environment source was suspected, and the connection has been established (Aitken et al., 2012).

We have established the Caenorhabditis elegans as an experimental model of M. avium subsp. hominissuis (M. avium) colonization and infection (Everman et al., 2015). It is wellaccepted that $M$. avium infects individuals by both respiratory and intestinal routes (Sangari et al., 2000; Babrak et al., 2015). Therefore, the establishment of a transmission model, that can be used to investigate infections acquired from the environment as well as from other hosts, is desirable. Since M. avium is a bacterium encountered in many environmental sources sharing common sites with $C$. elegans, we hypothesized that the nematode has the natural possibility of being infected by $M$. avium. Recent results demonstrated that $M$. avium can in fact infect C. elegans and invade the intestinal mucosal cells in a similar manner that it does in humans (Sangari et al., 2000; Babrak et al., 2015). The model developed takes advantage of the ability to genetically manipulate C. elegans and its environment.

With the establishment of this model, we now can ask questions related to transmission and "colonization" of host by $M$. avium. In this report we show that $M$. avium infection can be transmitted between nematode hosts and identified bacterial genes involved in the successful colonization or attachment to the epithelial mucosa of the new host.

\section{MATERIALS AND METHODS}

\section{Host Cells and C. elegans}

C. elegans strain $\mathrm{N} 2$ was maintained in monoxenic cultures with the addition of Escherichia coli strain OP50, and propagated on nematode growth medium (NGM) agar plates at $25^{\circ} \mathrm{C}$ as previously described by Brenner (1974). Human epithelial cells (HEp-2) were obtained from the American Type Culture Collection (ATCC, Manassas, VA, USA). HEp-2 cells were grown in RPMI-1640 medium supplemented with 10\% heat-inactivated fetal bovine serum (Gemini Bio-Products, West Sacramento, CA, USA) at $37^{\circ} \mathrm{C}$ in an atmosphere containing $5 \% \mathrm{CO}_{2}$.

\section{Bacteria}

All the experiments were carried out under biosafety level 2 containment (BSL2). E. coli strain OP50 was grown in Luria Bertani broth overnight, prior to inoculation of NGM plates at $37^{\circ}$ C. M. avium subsp. hominissius strain 104 , and M. avium subsp. hominissuis strain A5 were grown onto Middlebrook 7H10 agar supplemented with $10 \% \mathrm{w} / \mathrm{v}$ oleic acid, albumin, dextrose, and catalase (OADC, Hardy Diagnostics, Santa Maria, CA, USA) for 10 days at $37^{\circ} \mathrm{C}$. Bacteria lawn was established on agar plates to feed C. elegans. All the studies were carried out in biosafety level 2 laboratory, and the protocols have been reviewed and approved by the Biosafety Committee of the University.

\section{Construction and Screening of a Transposon Library}

$M$. avium mutants were obtained from the screening of a transposon library recently created in our laboratory (Rose and Bermudez, 2016). Briefly, M. avium subsp. hominissuis strain MAC A5 was transduced with the MycomarT7 phagemid at an MOI of 2 for $4 \mathrm{~h}$ at $37^{\circ} \mathrm{C}$. Aliquots of the transduction were plated onto Middlebrook 7H10 media containing $400 \mu \mathrm{g} / \mathrm{ml}$ of kanamycin. The library was screened by culturing HEp-2 cells in RPMI-1640 supplemented with 10\% heat-inactivated FBS in a 6-well tissue culture plate and adding to it a suspension containing 5,000 pooled clones from the M. avium A5 library for $45 \mathrm{~min}$ at $37^{\circ} \mathrm{C}$. After the $45 \mathrm{~min}$ incubation, the supernatant containing bacteria that did not adhere to the cell monolayer, was removed and added to a different monolayer of HEp2 cells for an additional $45 \mathrm{~min}$. The process was repeated 8 times for enrichment of clones that could not bind to the cells (without passage in culture medium) and the bacterial suspension of the last passage, containing clones that failed to bind to HEp- 2 cells, plated onto 7H10 agar. After 10 days, isolated colonies were obtained and tested individually for the 
ability to bind to HEp-2 cells using the described protocol (Bermudez and Young, 1994). Briefly, individual clones were incubated with $100 \%$ confluent HEp-2 cell monolayer for $1 \mathrm{~h}$ at $4{ }^{\circ} \mathrm{C}$. After the period, the supernatant was removed, the monolayers gently washed once with HBSS, and then the monolayers were lysed as previously described (Bermudez and Young, 1994). The number of adherent bacteria in each clone tested was compared to the adherence of the wildtype bacterium, and the clones which have a $80 \%$ or greater decrease in binding to the HEp-2 epithelial cells were selected. Clones impaired in binding were submitted to ligation-mediated PCR and DNA sequencing for identification of the location of the transposon, as previously described (Rose and Bermudez, 2016).

\section{Transmission Assay}

The transmission assay was performed by placing C. elegans, initially grown on a layer of $E$. coli OP50, on starvation media for 3 days $\left(25^{\circ} \mathrm{C}\right)$ and then removing the nematode and placing it on a lawn of $M$. avium A5, M. avium 104, or mutants $\left(1 \times 10^{5}\right.$ bacteria) for $4 \mathrm{~h}\left(25^{\circ} \mathrm{C}\right)$. After the period of time, C. elegans were removed out of the plate, the bacteria bound to the outside of the nematode body cleared as previously described (Everman et al., 2015). C. elegans (20 worms) were then placed onto an agar plate without bacteria for $2 \mathrm{~h}$ at $25^{\circ} \mathrm{C}$. In the next step, infected $C$. elegans were then removed from the agar using a platinum wire pick. Fresh, uninfected C. elegans, maintained without eating for 3 days, were transposed to the plate that at this point contained bacteria excreted from the previously removed $C$. elegans. After an additional $2 \mathrm{~h}$, the plates were washed in M9 saline (Everman et al., 2015) supplemented with $25 \mathrm{mM}$ of levamisole hydrochloride (SigmaAldrich) for paralysis and prevention of elimination or uptake of bacteria during the washes. C. elegans were then exposed to amikacin sulfate $(200 \mu \mathrm{g} / \mathrm{ml})$ for $30 \mathrm{~min}$ to kill all bacteria bound to the outside of the nematode body (Everman et al.,
2015), and subsequently washed twice in HBSS. To quantify bacteria ingested by $C$. elegans, suspensions of 10 nematodes were homogenized using a handheld motorized pot (VWR, Radnor, PA, USA) for $1 \mathrm{~min}$ in $0.1 \%$ triton $\mathrm{X}-100$. Then, samples were diluted in sterile water, plated onto Middlebrook 7H10 agar, and 10 days later the number of viable bacteria determined. As a control for the bacterial killing in the surface, C. elegans were placed and "rolled over" in a LB agar plate as well as a Middlebrook 7H10 agar plate. Plates were checked for mycobacterial growth for 2 months.

\section{Microscopy (Histology and Transmission Electron Microscopy)}

For histology worms were collected in M9 saline solution, washed twice in saline with centrifugation at $225 \times \mathrm{g}$ for $2 \mathrm{~min}$ to remove potential extracellular bacteria. Nematodes were fixed in $10 \%$ buffered formula for $5 \mathrm{~min}\left(24^{\circ} \mathrm{C}\right)$ and placed in melting agarose. Agarose-encased worms were embedded in resin and section mounted into glass slides. Specimens were stained with acid-fast stain and visualized.

\section{Transmission Electron Microscopy}

Worms collected in M9 solution were washed twice and the pellet was suspended in fixative buffer with $2.5 \%$ glutaraldehyde, $1 \%$ formaldehyde, and $0.1 \mathrm{M}$ sodium cacodylate. Sections were stained, dehydrated and visualized in the TEM facility of Oregon State University.

\section{Statistical Analysis}

Results reported represent the data obtained in at least two experiments performed independently \pm standard error. Analysis was done by using Graphpad Prism 6. The statistical significance of the binding assays were determined using the Student's $t$-test and by the ANOVA test. $p<0.05$ meaning statistical significance.

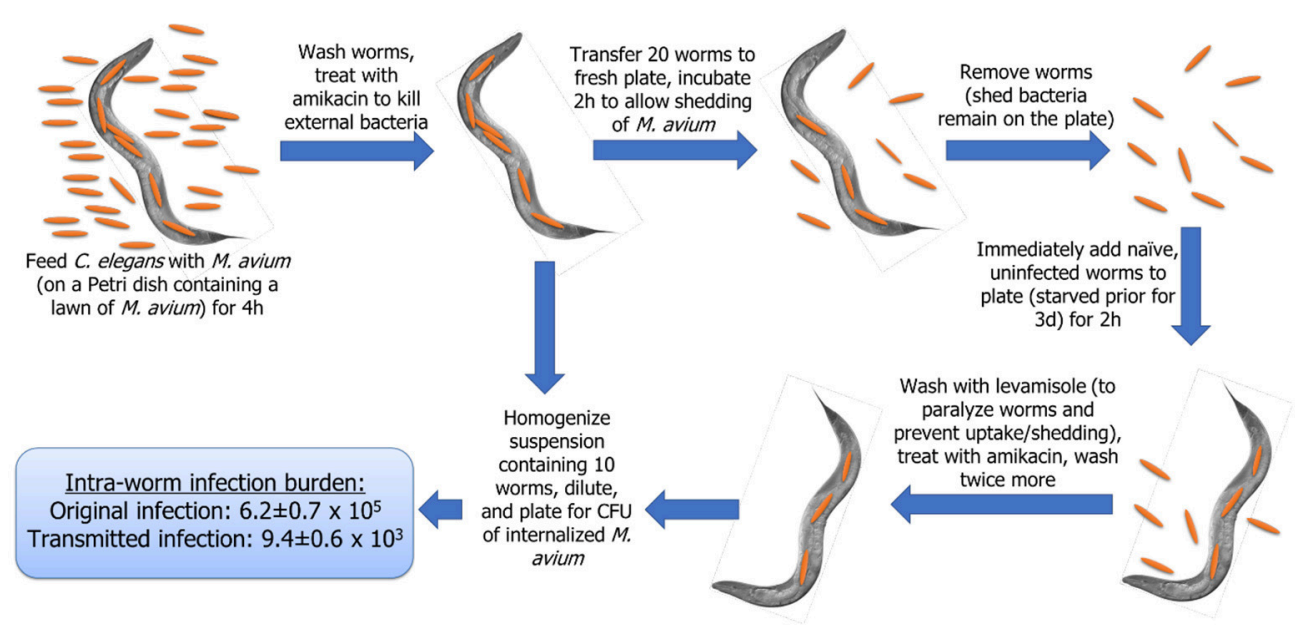

FIGURE 1 | Schematic representation of the model used. 


\section{RESULTS}

\section{Transmission of M. avium}

To determine whether $M$. avium could be transmitted from one host (infected) to another (naïve) host without prolonged passage in the environment, $M$. avium strain 104 was used to infect C. elegans orally (by seeding it on a plate) and then the nematode was transferred to another plate, this one without a lawn of bacteria, as described in section Materials and Methods and summarized in Figure 1.

After the elimination of bacteria by the first nematode hosts (infected), the C. elegans were again removed from the plate and substituted by uninfected, starving C. elegans (naïve). After $2 \mathrm{~h}$, previously uninfected nematodes were removed and their out-surface sterilized. The nematodes were then homogenized and plated for quantification of CFU. Some of the nematodes were prepared for histopathology and electron microscopy

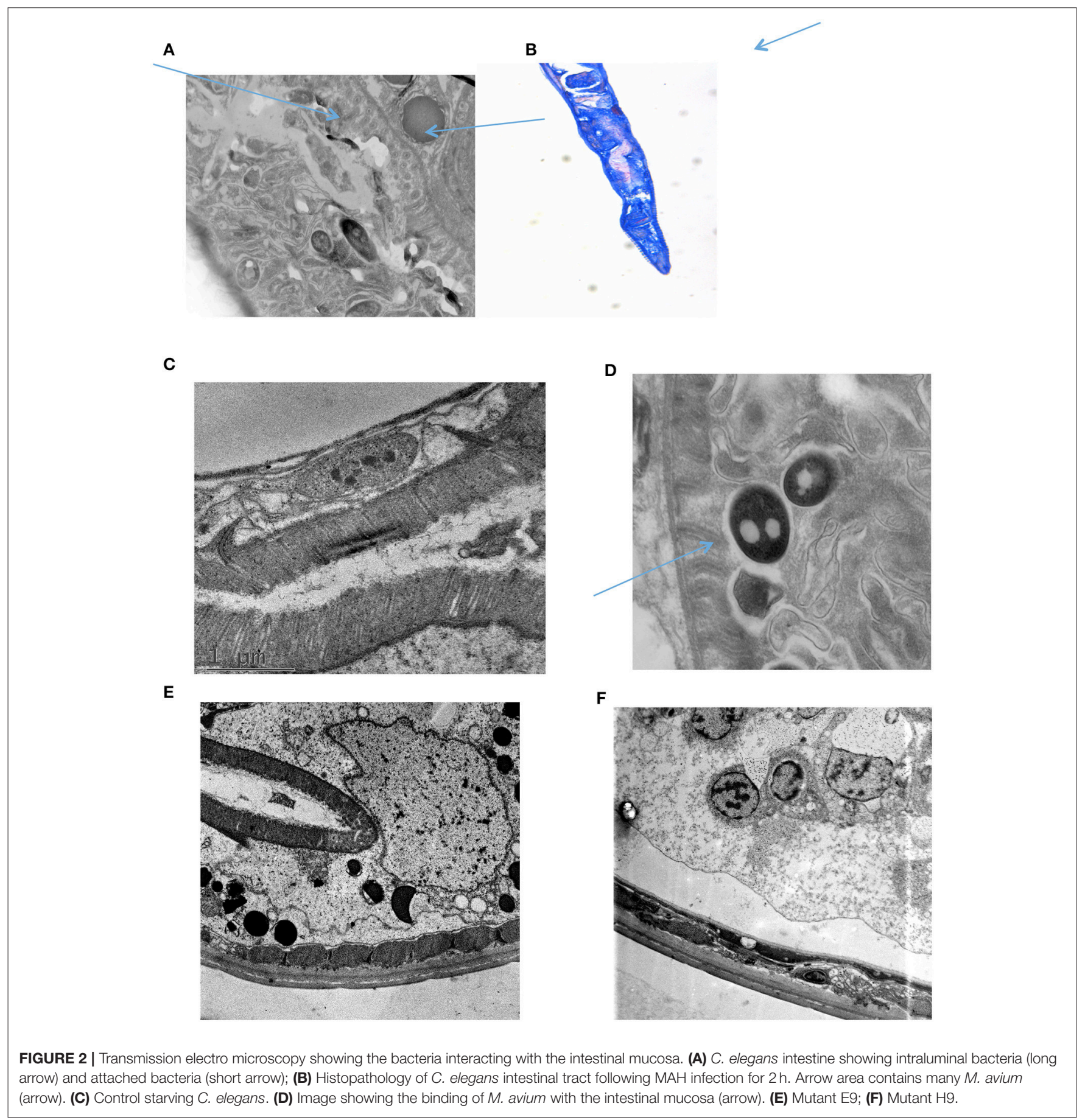


TABLE 1 | Transmission of M. avium strains 104 and A5 between C. elegans.

\begin{tabular}{lcc}
\hline M. avium strain & $\begin{array}{c}\text { \# Bacteria first } \\
\text { C. elegans }\end{array}$ & $\begin{array}{c}\text { \# Bacteria second } \\
\text { C. elegans }\end{array}$ \\
\hline 104 & $6.2 \pm 0.4 \times 10^{5}$ & $9.4 \pm 0.6 \times 10^{3}$ \\
A5 & $3.9 \pm 0.5 \times 10^{4}$ & $6.7 \pm 0.2 \times 10^{3}$ \\
\hline
\end{tabular}

The numbers represent the average of 2 experiments with 20 C. elegans (first host) and $20 \mathrm{C}$. elegans (second host) for each of the M. avium strains. The results of two experiments were very comparable.

as previously described (Everman et al., 2015). As shown in Figures $\mathbf{2 A , B}$, infection was transmitted from one C. elegans to another and the pathogen can interact with the intestinal mucosa. The infected C. elegans had $6.2 \pm 0.4 \times 10^{5}$ bacteria (MAH104) or $3.9 \pm 0.5 \times 10^{4}$ A5 strain in the intestines, while the initially naïve nematodes had an average of $9.4 \pm 0.6 \times 10^{3}(\mathrm{MAH})$ or 6.7 $\pm 0.2 \times 10^{3}$ (A5) bacteria in the intestinal lumen after exposed to bacteria (Table 1).

\section{Screening for Colonization Mutants}

It was then decided to select for clones that do not bind human epithelial cells to verify if those clones were also deficient in "colonizing" C. elegans. A pooled transposon library of 5,000 mutants was screened for impaired ability to bind to human HEp-2 epithelial cells. After eight serial infections with the same pooled library inoculum that was intended to enrich for clones that were impaired in binding, the final supernatant was removed from HEp-2 cells and plated. Ninety-two colonies from this enrichment were re-grown, and re-tested individually for their binding ability to HEp-2 cells. Forty-seven of the 92 clones showed a greater than $50 \%$ reduction in binding to HEp-2 cells compared with the wildtype bacterium. Thirtyseven clones were chosen for sequencing, and in 30 out of 37 clones the gene interrupted by the transposon was successfully identified (Table 2). From the 30 clones sequenced, 29 were unique gene sequences while one gene had a repetitive sequence. This information suggests that although we did not screen the library to saturation (library size of 100,000 clones) we already obtained repetitive genes in the screening, suggesting the gene importance for binding and colonization. Among the genes identified, there were 5 hypothetical proteins (all probable membrane proteins). The ontology of the class of protein encoded genes is show in Table 3. Two genes encoded for membrane proteins with homology to genes in M. tuberculosis, M. avium, and M. abscessus. Among the identified domains, one (FAM53) is known to bind to eukaryotic cell membrane. A TetR transcription regulator, that likely influences the expression of surface-related proteins, was also identified. Four proteins do not have the function known, and with the exception of one of them (clone G12), which has homology to a flagella protein, do not have known motifs. An oxidoreductase, monooxygenases (2 of them), and aminotransferase were enzymes linked with the phenotype. Out of them, we have evidence for an oxidoreductase from M. avium subsp. paratuberculosis to be important for the entry of the bacteria in epithelial cells (Alonso-Hearn et al., 2008), by participating on the folding of invasion-related proteins.
The clone B2 has MAVA5_06340 gene interrupted. Sequence information tells that the gene exists in both A5 and 104 strains as an isolated gene. The E9 clone (MAV_01195) is also an isolated gene. The clone G12 (MAV_15020) is the gene in the end of a two-gene operon. The clones H9 and B5, the genes interrupted are in the middle of the operon, what require complementation to rule out the phenotype being due to a downstream gene.

\section{Evaluation of the M. avium Mutants for Colonization of $C$. elegans}

To determine if the clones with impaired ability to bind to human mucosal epithelial cells were also deficient in transmission between nematodes, we infected C. elegans orally with each, the wildtype bacteria and the binding-deficient clones. We also determined the growth of bacterial clones on Middlebrook 7H9 and 7H10 media. All of the mutants used (B5, E2, H9, G12, and E9) grew in a comparable fashion to the wildtype bacterium (MACA5).

As shown in Figures 2A-D, M. avium can infect C. elegans with attachment and invasion of the intestinal tract mucosa. Based on that information, we selected 5 mutants that showed impairment to adhere to human epithelial cells in vitro, and one positive control mutant (4B2, deficient in GPL) that does not adhere to epithelial cells efficiently. The results obtained in C. elegans confirmed the deficiency observed of the clones to attach to human epithelial cells (Table 4). All five tested clones showed impairment of infection that were statistically significant.

\section{DISCUSSION}

M. avium infection of the lung is believed to be always acquired from an environmental source (Griffith et al., 2007; Adjemian et al., 2012; Ringshausen et al., 2016). Recent events suggested that it may not be the case (Vaghaiwalla et al., 2014), and environmental mycobacteria could be transmitted from host-tohost in unidentified occasions. Assuming that it was the case, what makes it difficult to establish the epidemiologic link is that the bacterium could in fact infect the host weeks or even months before the clinical disease would develop and could be diagnosed and linked to the source.

In this work we used the nematode C. elegans to establish a transmission model, in which an $M$. avium strain eliminated from one $C$. elegans can be transmitted, without remaining in the environment for an extended period of time, to a "naïve" C. elegans and ultimately establish infection. Once it was demonstrated that the $C$. elegans model was feasible to study transmission, we screened a transposon library of $M$. avium for clones that had deficiency in attaching to human mucosal epithelial cells, and then used the selected mutants in the C. elegans assay. All the five mutants selected were shown to have impaired ability to bind to the intestinal mucosa of C. elegans.

The initial indication is that the screening in vitro for deficiency in binding to epithelial cells seems to provide results that can be reproduced in other models. In despite of the fact that we only examined five adherence-deficient out of 30 clones using the C. elegans model, the results obtained suggest that the system 
TABLE 2 | Clones deficient in rapid attachment to HEp-2 cells.

\begin{tabular}{|c|c|c|c|c|}
\hline Clone & Reduction from WT (\%) ${ }^{a}$ & Gene & Encoded protein & Homologs ${ }^{b} /$ location $\mathrm{Tn}^{c}$ \\
\hline E4 & 98.2 & MAVA5_06540 & Dihydropteroate synthase & MAV_1352, Rv1207, MAB_1345/58 n C-terminus \\
\hline D6 & 95.4 & MAVA5_10295 & 4-hydroxyacetophenone monooxygenase & MAV_1795, MAB_4476c 104 n N-terminus \\
\hline $\mathrm{H} 8$ & 93.7 & MAVA5_14105 & Methylmalonyl-CoA mutase & MAV_3277, Rv1493, MAB_2711c/110 n N-terminus \\
\hline $\mathrm{H} 4$ & 92.2 & MAVA5_09730 & Hydrolase & MAV_2243, Rv2223c, MAB_1919/106 n N-terminus \\
\hline E6 & 91.5 & MAVA5_15805 & Acyl-CoA dehydrogenase & MAV_3616, Rv2724c, MAB_3040c/120 n N-terminus \\
\hline G10 & 89.3 & MAVA5_04860 & Major facilitator transporter & MAV_1023, Rv2456c, MAB_3449c/146 n N-terminus \\
\hline D10 & 88.3 & MAVA5_03005 & Alcohol dehydrogenase & MAV_0705, Rv0761c, MAB_4560/middle of gene \\
\hline H3 & 88.0 & MAVA5_15805 & Acyl-CoA dehydrogenase & MAV_3616, Rv2724c, MAB_3040c/ 101 n N-terminus \\
\hline D7 & 87.9 & MAVA5_15910 & $\mathrm{N}$-acetylglutamate synthase & MAV_3638, Rv2747, MAB_3072/ 106 n N-terminus \\
\hline D8 & 86.6 & MAVA5_21725 & Succinate-semialdehyde dehydrogenase & MAV_4936, Rv0234c, MAB_3471/ 121 n N-terminus \\
\hline D5 & 79.8 & MAVA5_10295 & 4-hydroxyacetophenone monooxygenase & MAV_1795, MAB_4476c/ $105 \mathrm{n} \mathrm{N}$-terminus \\
\hline B5 & 76.2 & MAVA5_11640 & Oxidoreductase & MAV_2766/Middle of gene \\
\hline H9 & 73.7 & MAVA5_08165 & Membrane protein & MAV_1726, Rv2446c, MAB_1605c/ middle of gene \\
\hline F12 & 73.6 & MAVA5_12410 & Hypothetical protein & MAV_2925, Rv1787/ middle of gene \\
\hline E9 & 65.8 & MAVA5_01195 & Aminotransferase & MAV_0250, Rv3772, MAB_0220/210 n N-terminus \\
\hline E2 & 64.6 & MAVA5_08520 & Acetyl hydrolase & MAV_1798, Rv2385, MAB_2076/121 n N-terminus \\
\hline E12 & 63.0 & MAVA5_11775 & Hypothetical protein & None/96 n N-terminus \\
\hline G12 & 59.7 & MAVA5_15020 & Hypothetical protein & MAV_3472/111 n N-terminus \\
\hline B2 & 58.4 & MAVA5_06340 & Hypothetical protein & MAV_1314, Rv1174c, MAB_2488c/middle of gene \\
\hline F9 & 54.5 & MAVA5_04920 & Hypothetical protein & None/105 n N-terminal \\
\hline C2 & 54.4 & MAVA5_22565 & Monooxygenase & MAV_5206/42 n C-terminus \\
\hline C5 & 54.2 & MAVA5_02065 & Inhibition of morphological differentiation protein & MAV_0469, Rv3661, MAB_0431c/110 n N-terminus \\
\hline
\end{tabular}

${ }^{a}$ This value was calculated from the CFU recovered bound to and/or invaded in the epithelial cells between the average of four wildtype samples with the respective clone.

${ }^{b}$ The respective clone was compared with the type strains M. avium subsp. hominissuis 104, M. tuberculosis H37Rv, and M. abscessus subsp. abscessus 19977 to determine homology.

cLocation of transposon: Nucluotides from C- or N-terminus.

TABLE 3 | Ontology of the 30 genes sequenced that were associated with binding and/or colonization of the epithelial mucosa.

\begin{tabular}{lc}
\hline Pathways/groups of genes & $\begin{array}{c}\text { Number of genes associated with } \\
\text { phenotype }\end{array}$ \\
\hline Metabolic pathways & 8 \\
Signaling & 1 \\
Transport Proteins & 2 \\
Transcription regulators & 1 \\
Membrane proteins & 5 \\
Folate, DNA synthesis & 1 \\
Flavoprotein, energy & 1 \\
Fatty acid synthesis & 6 \\
\hline
\end{tabular}

can be employed to study transmission and "colonization" and perhaps, in the future, how to prevent it.

The second important finding is that the model confirmed the possibility that in many occasions $M$. avium may be acquired
TABLE 4 | Binding of wild type and mutant strains to C. elegans intestine.

\begin{tabular}{lccc}
\hline Bacteria strain/clone & CFU/plate & $\begin{array}{c}\text { CFU/worm intestine/ } \\
\text { 30 } \text { min }^{\mathbf{a}}\end{array}$ & $\boldsymbol{P}^{\text {-value }} \mathbf{b}^{\mathbf{b}}$ \\
\hline MAH 104 & $6.4 \times 10^{5}$ & $4.7 \pm 0.6 \times 10^{4}$ & - \\
MAH A5 & $5.9 \times 10^{5}$ & $3.0 \pm 0.5 \times 10^{4}$ & - \\
B5 & $5.6 \times 10^{5}$ & $2.2 \pm 0.4 \times 10^{3}$ & $<0.05$ \\
B2 & $6.4 \times 10^{5}$ & $5.7 \pm 0.4 \times 10^{2}$ & $<0.02$ \\
H9 & $5.2 \times 10^{5}$ & $2.1 \pm 0.4 \times 10^{2}$ & $<0.01$ \\
G12 & $5.3 \times 10^{5}$ & $2.7 \pm 0.6 \times 10^{2}$ & $<0.02$ \\
E9 & $6.1 \times 10^{5}$ & $1.5 \pm 0.8 \times 10^{2}$ & $<0.01$ \\
4B2 $(\Delta G$ GL) & $5.9 \times 10^{5}$ & $1.4 \pm 0.3 \times 10^{3}$ & $<0.05$
\end{tabular}

a 10 C. elegans were exposed to MAH/excretion of $20 \mathrm{C}$. elegans.

${ }^{b}$ The calculation of statistical significance was carried out using the ANOVA test. $P<0.05$ was considered significant.

from a living source, such as an infected patient with chronic pulmonary condition, instead of from the outside environment. Expending the model to determine the virulence determinants 
in the pathogen which are associated with infection can be advantageous over the current available systems. Furthermore, C. elegans is a genetically treatable host, and mutations can be obtained in the genes with potential to be associated with mechanisms of host innate defense.

The final finding of this work is that genes were identified in association to binding, and, when inactivated, resulted in significant decrease of adherence to human respiratory epithelial cells as well as the ability of the mutant clone to colonize C. elegans. A number of the identified genes encode for proteins of unknown function, while other genes identified encode for enzymes involved in the synthesis of cell wall or surface structures, fatty acid synthesis, metabolic pathways, and transporters among others (Tables 2, 3). The latter may be related with the assemble of the outer surface of the bacterium. The consequent conclusion is that the pathogen seems to utilize several molecules to interact with the surface of the epithelial cells. It would be important to determine if there is a hierarchy of molecules that follow the attachment or whether the process is indiscriminate or partially redundant. That information may have important consequences to the development of a strategy to diminish or prevent colonization.

\section{REFERENCES}

Adjemian, J., Olivier, K. N., Seitz, A. E., Holland, S. M., and Prevots, D.R. (2012). Prevalence of nontuberculous mycobacterial lung disease in U.S. Medicare Beneficiaries. Am. J. Respir. Crit. Care Med. 185, 881-886. doi: 10.1164/rccm.201111-2016OC

Aitken, M. L., Limaye, A., Pottinger, P., Whimbey, E., Goss, C. H., Tonelli, M. R., et al. (2012). Respiratory outbreak of Mycobacterium abscessus subspecies massiliense in a lung transplant and cystic fibrosis center. Am. J. Respir. Crit. Care Med. 185, 231-232. doi: 10.1164/ajrccm.185.2.231

Alonso-Hearn, M., Patel, D., Danelishvili, L., Meunier-Goddik, L., and Bermudez, L. E. (2008). The Mycobacterium avium subsp. paratuberculosis MAP3464 gene encodes an oxidoreductase involved in invasion of bovine epithelial cells through the activation of host cell Cdc42. Infect. Immun. 76, 170-178. doi: 10.1128/IAI.01913-06

Babrak, L., Danelishvili, L., Rose, S. J., Kornberg, T., and Bermudez, L. E. (2015). The environment of "Mycobacterium avium subsp. hominissuis" microaggregates induces synthesis of small proteins associated with efficient infection of respiratory epithelial cells. Infect Immun. 83, 625-636. doi: 10.1128/IAI.02699-14

Bermudez, L. E., and Young, L. S. (1994). Factors affecting invasion of HT-29 and HEp-2 epithelial cells by organisms of the Mycobacterium avium complex. Infect. Immun. 62, 2021-2028.

Brenner, S. (1974). The genetics of Caenorhabditis elegans. Genetics 77, 71-94.

Everman, J. L., Ziaie, N. R., Bechler, J., and Bermudez, L. E. (2015). Establishing Caenorhabditis elegans as a model for Mycobacterium avium subspecies hominissuis infection and intestinal colonization. Biol. Open 4, 1330-1335. doi: 10.1242/bio.012260

Falkinham, J. O. III. (2009). Surrounded by mycobacteria: nontuberculous mycobacteria in the human environment. J. Appl. Microbiol. 107, 356-367. doi: 10.1111/j.1365-2672.2009.04161.x

Faria, S., Joao, I., and Jordao, L. (2015). General overview on nontuberculous mycobacteria, biofilms, and human infection. J. Pathog. 2015:809014. doi: 10.1155/2015/809014

Griffith, D. E., Aksamit, T., Brown-Elliott, B. A., Catanzaro, A., Daley, C., Gordin, F., et al. (2007). An official ATS/IDSA statement: diagnosis, treatment, and prevention of nontuberculous mycobacterial diseases. Am. J. Respir. Crit. Care Med. 175, 367-416. doi: 10.1164/rccm.200604-571ST
The limitations of the current study is that C. elegans system appears to work as initial screening, but the phenotype should be confirmed in a more complex system, as done with the strain 4B2.

In summary, we established a model of host-to-host transmission of NTM (M. avium), and identified genes associated with attachment of the bacterium to the host mucosa. Future work will explore the model in the attempt to identify strategies to prevent colonization.

\section{AUTHOR CONTRIBUTIONS}

LB performed experiments, wrote the paper, principal investigator. SR performed experiments, wrote the paper. JE and NZ perform experiments.

\section{ACKNOWLEDGMENTS}

We are thankful for the editorial help from Beth Chamblin and Carolyn Cowan.

The work was supported by the grant AI043199 from the National Institute of Allergy and Infectious Diseases and by the Microbiology Foundation of San Francisco, CA.

Jamal, W., Salama, M. F., Al Hashem, G., Rifaei, M., Eldeen, H., Husain, E. H., et al. (2014). An outbreak of Mycobacterium abscessus infection in a pediatric intensive care unit in Kuwait. Pediatr. Infect. Dis. J. 33: e67-e70. doi: 10.1097/INF.0000000000000071

Namkoong, H., Kurashima, A., Morimoto, K., Hoshino, Y., Hasegawa, N., Ato, M., et al. (2016). Epidemiology of pulmonary nontuberculous mycobacterial disease, Japan(1). Emerg. Infect. Dis. 22, 1116-1117. doi: 10.3201/eid2206.151086

Primm, T. P., Lucero, C. A., and Falkinham, J. O. (2004). Health impacts of environmental mycobacteria. Clin. Microbiol. Rev. 17, 98-106. doi: 10.1128/CMR.17.1.98-106.2004

Ringshausen, F. C., Wagner, D., de Roux, A., Diel, R., Hohmann, D., Hickstein, L., et al. (2016). Prevalence of nontuberculous mycobacterial pulmonary disease, Germany, 2009-2014. Emerg. Infect. Dis. 22, 1102-1105. doi: 10.3201/eid2206.151642

Rose, S. J., and Bermudez, L. E. (2016). Identification of bicarbonate as a trigger and genes involved with extracellular DNA export in mycobacterial biofilms. mBio 7:e01597-16. doi: 10.1128/mBio.01597-16

Sangari, F. J., Goodman, J., and Bermudez, L. E. (2000). Mycobacterium avium enters intestinal epithelial cells through the apical membrane, but not by the basolateral surface, activates small GTPase Rho and, once within epithelial cells, expresses an invasive phenotype. Cell. Microbiol. 2, 561-568. doi: 10.1046/j.1462-5822.2000.00080.x

Vaghaiwalla, T., Satahoo, S. S., Zarifa, R., Dauer, M., Davis, J. S., Dearmas, D., et al. (2014). Mycobacterium abscessus infection in a burn intensive care unit patient. Surg. Infect. 15, 847-849. doi: 10.1089/sur.2014.052

Conflict of Interest Statement: The authors declare that the research was conducted in the absence of any commercial or financial relationships that could be construed as a potential conflict of interest.

Copyright (c) 2018 Bermudez, Rose, Everman and Ziaie. This is an open-access article distributed under the terms of the Creative Commons Attribution License (CC $B Y)$. The use, distribution or reproduction in other forums is permitted, provided the original author(s) and the copyright owner are credited and that the original publication in this journal is cited, in accordance with accepted academic practice. No use, distribution or reproduction is permitted which does not comply with these terms. 